\title{
Development and validation of a model of motor competence in children and adolescents
}

\author{
Carlos Luz $^{\mathrm{a}, \mathrm{b}, *}$, Luis P. Rodrigues ${ }^{\mathrm{d}, \mathrm{e}}$, Gabriela Almeida $^{\mathrm{c}}$, Rita Cordovil $^{\mathrm{f}}$ \\ a Laboratory of Motor Behavior, Faculdade de Motricidade Humana, Universidade de Lisboa, Portugal \\ ${ }^{\mathrm{b}}$ Escola Superior de Educação de Lisboa, Instituto Politécnico de Lisboa, Portugal \\ c Faculdade de Ciências da Saúde, Universidade Fernando Pessoa, Portugal \\ d Escola Superior de Desporto e Lazer, Instituto Politécnico de Viana do Castelo, Portugal \\ e CIDESD, Portugal \\ ${ }^{\mathrm{f}}$ Laboratory of Motor Behavior, CIPER, Faculdade de Motricidade Humana, Universidade de Lisboa, Portugal
}

\section{A R T I C L E I N F O}

\section{Article history:}

Received 23 December 2014

Received in revised form 15 June 2015

Accepted 2 July 2015

Available online 10 July 2015

\section{Keywords:}

Motor competence

Physical education

Quantitative instruments

Locomotor

Manipulative

Stability

\begin{abstract}
A B S T R A C T
Objectives: This study was aimed at developing a quantitative model to evaluate motor competence (MC) in children and adolescents, to be applicable in research, education, and clinical contexts. Design: Cross-sectional.

Methods: A total of 584 children (boys $n=300$ ) with ages between 6 and 14 years were assessed using nine well known quantitative motor tasks, divided into three major components (stability, locomotor and manipulative). Structural equation modelling through EQS 6.1 was used to find the best model for representing the structural and measurement validity of MC.

Results: The final MC model was composed by three latent factors closely related with each other. Each factor was best represented by two of the initial three motor tasks chosen. The model was shown to give a very good overall fit $\left(\chi^{2}=12.04, p=.061 ; \mathrm{NFI}=.982 ; \mathrm{CFI}=.991\right.$; $\left.\mathrm{RMSEA}=.059\right)$.

Conclusions: MC can be parsimoniously represented by six quantitative motor tasks, grouped into three interrelated factors. The developed model was shown to be robust when applied to different samples, demonstrating a good structural and measurement reliability. The use of a quantitative protocol with few, simple to administer and well known, motor tasks, is an important advantage of this model, since it can be used in several contexts with different objectives. We find it especially beneficial for physical educations teachers who have to regularly assess their students.
\end{abstract}

(C) 2015 Sports Medicine Australia. Published by Elsevier Ltd. All rights reserved.

\section{Introduction}

In the last two decades a growing body of evidence suggests that early Motor Competence (MC) is of paramount importance for developing an active and healthy lifestyle. ${ }^{1} \mathrm{MC}$ is used as a global term to describe a person's ability to be proficient on a wide range of motor acts or skills. ${ }^{2}$ This ability has been described in the literature also as motor coordination, motor performance, or motor proficiency. In the initial phases of motor development, children's $\mathrm{MC}$ involves the mastery of fundamental motor skills that are the foundations for the mastery of specialized motor skills. It has been reported that physical activity, ${ }^{3,4}$ cardiorespiratory fitness, 5,6 physical fitness, ${ }^{7}$ and perceived physical competence, ${ }^{8,9}$ have positive effects and associations with MC, as well as an inverse association

\footnotetext{
* Corresponding author.

E-mail address: carlosmiguelluz@gmail.com (C. Luz).
}

with weight status ${ }^{10}$ in children and adolescents. This has provided emerging evidence to the theoretical model proposed by Stodden and colleagues. ${ }^{1}$

MC as a theoretical construct is considered to be subdivided into locomotor (e.g., leaping, galloping or vertical jump), stability (e.g., dynamic and static balance) and manipulative (e.g., catching, throwing and kicking $)^{11}$ proficiency. However, this structure is not always reflected in research and/or clinical settings where MC constitutes the subject of interest. Several standardized tests (e.g., TGMD, KTK), and a number of different non-standardized protocols, ${ }^{12,13}$ found in the literature, are deemed to evaluate MC but do not follow the theoretical MC construct. For example, the TGMD does not evaluate stability and the KTK does not evaluate manipulative proficiency. Furthermore, most instruments and protocols are restricted to a specific age, or narrow age-range, either due to the developmental restricted age window of the motor tasks, or to the nature of the used scoring procedures (quantitative or qualitative). 
This great discrepancy between the accepted theoretical construct of MC and its application in research and/or clinical settings shows the lack of a robust conceptual and working model of MC that could be successfully used in different settings and developmental ages. To our knowledge, no studies have been presented that validate the theoretical MC model structure using the original three categories. Some studies have used structural equation modelling or confirmatory factor analysis techniques to look for the structural validity of instruments (e.g., M-ABC, ${ }^{14}$ TGMD $^{15}$ ) but the instruments themselves were not in full agreement with the theoretical MC model.

The main objective of this study was to establish a working developmental model of MC, based on three domains (locomotor, stability, and manipulative) of the theoretical construct of MC. We hypothesized that each of these three categories is represented by age independent significant motor tasks that can be objectively measured (product).

To achieve this purpose we have assessed children using several motor tasks representative of each MC category in the literature, and worked with the data to find a representative and parsimonious model of MC using specific Structural Equations Modelling (SEM) techniques.

\section{Methods}

A total of 584 children ( 300 males), aged 6 to 14 years $(M=10.60$, $\mathrm{SD}=2.40$ ), participated in this study. Children were randomly selected from public Portuguese schools and had no known learning disabilities or pre-existing motor limitations. A local ethics committee approval was obtained and parents provided written informed consent. Two Physical Education (PE) teachers with 10 years of experience were trained to collect the data in regular scheduled classes (each teacher always assessed the same group of tasks).

Three tests for each MC category (stability, locomotor, and manipulative $)^{11}$ were selected from the most used protocols and instruments in the motor development literature. Inclusion criteria were being quantitative (product-oriented) motor tests without a marked developmental (age) ceiling effect, and of feasible execution.

Stability tests were: (a) balance beams ${ }^{16}$-walking backwards on three balance beams with $3 \mathrm{~m}$ in length but of decreasing widths $(6,4.5$ and $3 \mathrm{~cm})$. Each participant had three attempts per beam and each attempt had the maximum score of 8 points. The total score was given by the sum of points in the three balance beams (72 total possible points); (b) Shifting platforms ${ }^{16}$-moving sideways for 20 s using two wooden platforms $(25 \mathrm{~cm} \times 25 \mathrm{~cm} \times 2 \mathrm{~cm})$. Each successful transfer from one platform to the other was scored with two points (one point for each step). Participants were given two trials and only the best score was considered; (c) jumping laterally ${ }^{16}$-jumping sideways with two feet together over a wooden beam as fast as possible for $15 \mathrm{~s}$. Each correct jump scored 1 point and the best result over two trials was considered. Locomotor tests were: (a) hopping on one leg over an obstacle ${ }^{16}$-jumping over a stack of foam blocks $5 \mathrm{~cm}$ high with one foot, reaching the floor with the same foot. After a successful attempt with each foot, the height was increased by adding one foam block. Participants received three, two or one point(s) for each successful performance on the first, second or third trial, respectively. Therefore, each child had three attempts at each height and on each foot. The testing was stopped when a height trial was not successfully completed with both feet. The total score was given by the sum of points at all the heights; (b) shuttle run (SHR) ${ }^{17}$-running at maximal speed to a line placed $10 \mathrm{~m}$ apart, picking up a block of wood, running back and placing it on or beyond the starting line. Then running back to retrieve the second block and carry it back across the finish line. The final score was the best time of the two trials; (c) standing long jump (SLJ) $)^{18}$-jumping with both feet simultaneously as far as possible. The final score (the better of 2 attempts) was the distance (in $\mathrm{m}$ ) between the starting line and the back of the heel at landing closest to the line. Manipulative tests were: (a) wall toss test ${ }^{19}$-throwing a tennis ball with an overarm action against the wall ( $2 \mathrm{~m}$ distance), attempting to catch it with both hands over $30 \mathrm{~s}$. The final score was given by the better result (number of catches) in 2 attempts; (b) throwing velocity ${ }^{20}$-throwing a baseball (circumference: $22.86 \mathrm{~cm}$; weight: $142 \mathrm{~g}$ ) at a maximum speed against a wall using an overarm action. Three trials were performed, and the final score was given by the best result; and (c) kicking velocity ${ }^{20}$-kicking a soccer ball no. 4 (circumference: $64 \mathrm{~cm}$, weight: $350 \mathrm{~g}$ ) at a maximum speed against a wall using a kicking action. Three trials were performed, and the final score was given by the best result. Ball peak velocity was measured with a Pro II Stalker Radar Gun in both tests.

Participants completed a general warm-up before the beginning of the tests. Then, groups of five students were evaluated in the same task order. Participants observed a demonstration of the proficient technique and had the opportunity to experiment with each task one time before their performance. Motivational feedback was given; however no verbal feedback on skill performance was provided. In the throwing/kicking tasks, children were instructed to throw/kick the ball as fast as they could.

In order to assess the plausibility and validity of our theoretically driven MC model, we used a special multi-group confirmatory factor analysis, known as stack model. ${ }^{21}$ In the first two steps of this procedure, the full sample (584 subjects) was randomly split in half maintaining the sex proportionality. The first half (292 subjects) was used as a calibration sample (to set the initial best model to entail MC according to the theoretical framework), and the second as a validation sample, used to assure that the previous chosen model (factors and loading items) was able to reproduce every other data.

On the third phase (cross validity) we formally tested for measure and structural invariance between the two split halves. To test for measure invariance, the formal structure from the calibration sample was imposed on the validation sample while all parameters were left free. Using a more restricted approach (tight cross validation), structural invariance was also imposed to the validation sample, with all parameters constrained to the calibration model values.

The absolute fit of the models (individual and multi-group analysis) was evaluated using the Satorra and Bentler scaled chi-square $\left(\chi^{2}\right)$ (1994) with correction for non-normality, while the relative fit was assessed using the comparative fit index (CFI), the normed fit index (NFI), and the goodness of fit index (GFI). For these indices, values over .95 and up to 1.0 are deemed indicative of a good fit.

The root mean square error of approximation (RMSEA) and respective confidence intervals $(\mathrm{CI})$ were used for evaluating how well the model-implied reproduced the variance-covariance matrix of the data, keeping in mind that RMSEA values as low as .06 represent a good fit to the model. ${ }^{21-23}$ EQS Lagrange Multiplier Tests (LMT) for adding and deleting parameters were interpreted within the theoretical framework for each model tested in the calibration phase, and alterations made accordingly. Variables were considered for deletion when LMT suggested that such procedure resulted in a significant improvement of the model fit. Each consecutive model was compared with the previous using the chisquare and degrees of freedom change, and was only retained when this comparison showed statistical significance. All analyses were conducted using the EQS 6.1 computer program (Multivariate Software, Inc.). 
Table 1

Indices for evaluating goodness of fit of models in different phases.

\begin{tabular}{|c|c|c|c|c|c|}
\hline & $\chi^{2}$ & $p$ & NFI & CFI & RMSEA \\
\hline \multicolumn{6}{|c|}{ Phase 1 -Calibration sample $(n=292)$} \\
\hline Model 1 (9 variables) & $159.39(24 d f)$ & .000 & .881 & .896 & .139 \\
\hline Model 2 (8 variables) & $134.31(18 d f)$ & .000 & .880 & .894 & .149 \\
\hline Model 3 (7 variables) & $96.69(11 d f)$ & .000 & .897 & .907 & .164 \\
\hline Model 4 (6 variables) & $12.04(6 d f)$ & .061 & .982 & .991 & .059 \\
\hline \multicolumn{6}{|c|}{ Phase 2 -Validation sample $(n=292)$} \\
\hline Model 4 (6 variables) & $12.15(6 d f)$ & .058 & .986 & .993 & .059 \\
\hline \multicolumn{6}{|c|}{ Phase 3-Multi-group analysis $(n=584)$} \\
\hline Measurement invariance & $24.20(12 d f)$ & .019 & .984 & .992 & .042 \\
\hline Structural invariance & $30.44(15 d f)$ & .010 & .980 & .990 & .042 \\
\hline
\end{tabular}

\section{Results}

In the calibration phase (phase 1) the initial formulation of the MC model was set according to the theoretical formulation with three factors (stability, locomotor, and manipulative), and three possible items (motor skill tasks) accounting (loading) on each factor. Departing from this theoretical model, we examined the solution according to the significance of the loading coefficients, $R^{2}$ values of the variables equations, and indices of overall and relative fit ( $\chi^{2}$, CFI, NFI and RMSEA). EQS Lagrange Multiplier tests for adding and deleting parameters were used to improve the model fit, according to theoretical interpretation. As a result of this procedure, three variables (hopping on one leg over an obstacle, walking on a balance beam, and tossing and catching a ball) were consecutively dropped from the original model, resulting in a final model of MC with six motor tasks (jumping laterally, shifting platforms, SHR, SLJ, throwing velocity, kicking velocity) and three correlated factors showing a very good overall fit $\left(\chi^{2}=12.04, p=.061 ; \mathrm{NFI}=.982\right.$; $\mathrm{CFI}=.991$; RMSEA $=.059 ; \mathrm{CI}(\mathrm{RMSEA})=(000-.106)$. This final standardized solution is shown in Fig. 1, and the fit indexes values for the four consecutive models tested can be seen in Table 1 .

In the second step (validation phase), data from the second half of the sample was tested using the final specified model from the calibration sample. Overall indices showed a very good adjustment of this model to the data (see Table 1), similar to the one found in the validation sample.

In the third step (cross validation phase), in order to test for the cross validity of the model we formally tested for measure and structural invariance between the two split halves. To test for measure invariance, the formal structure from the calibration model 4 sample was imposed on the validation sample while all parameters were left free. Indices $\left(\chi^{2}=24.20, p=.019 ; \mathrm{NFI}=.984 ; \mathrm{CFI}=.993\right.$; RMSEA $=.059)$ for the overall fit of this multi-group model were good (see Table 1). Using a more restricted approach (tight cross validation), structural invariance was also imposed to the validation sample, with all parameters constrained to the calibration model 4 loading values. Final results continued to show a good overall fit $\left(\chi^{2}=30.44, p=.010 ; \mathrm{NFI}=.980 ; \mathrm{CFI}=.990 ; \mathrm{RMSEA}=.042\right)$, and the formal testing for differences between the imposed parameter's values showed no significant values. So, the solution found for the calibration sample (model 4 ) showed a very good adjustment to the other half of the data, proving its validity for interpreting the MC model.

\section{Discussion}

The aim of this study was to establish a model of MC, based on a theoretically structure divided into locomotor, stability, and manipulative domains. In this endeavor, quantitative (productoriented) motor test protocols without a developmental (age) ceiling effect, and of feasible execution, were used. Our purpose when selecting only product-oriented tests was to ensure an objective evaluation and a good sensitivity to discriminate among competence levels across ages. ${ }^{20}$

The use of SEM for testing this specific model is of great utility since it allows to work from the data for reaching a final solution for a MC structure that represents well the communality (represented by the covariance) and the unique characteristics (non-explained covariance) of tests (items) and categories (factors). The overall adjustment indices, along with the individual coefficients for the paths involved (factor-item; factor-factor) provides a rationale for including or excluding each item (test), or factor (category), or path (representativeness of the tests to mark a category), to a better representation of the full model.

In the validation phase our results confirmed the existence of three latent factors representing the stability (shifting platform and jumping laterally), locomotor (SHR and SLJ), and manipulative (moving platform and jumping laterally) categories of MC, each one best represented by two of the initial three motor tasks chosen. These three factors show to reproduce very well three distinctive aspects of MC, as proved by the inexistence of any change

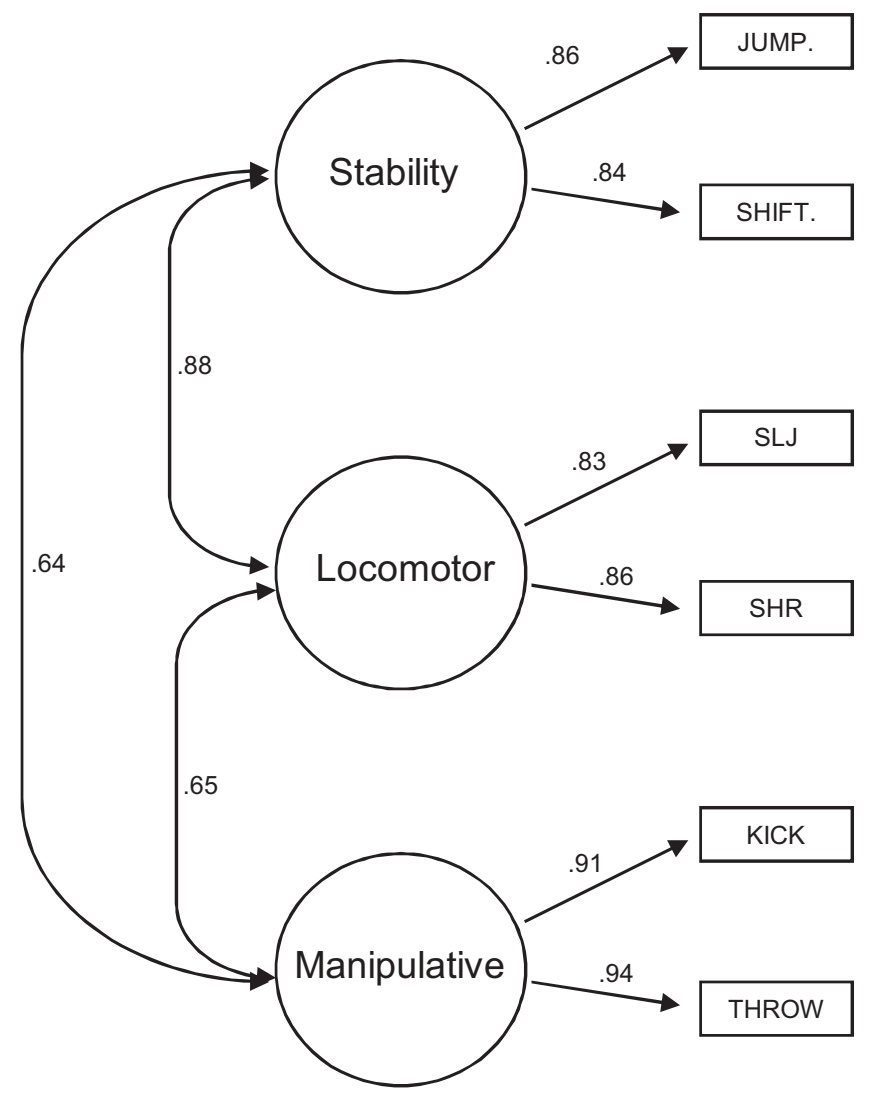

Fig. 1. Path diagram of the model for motor competence with completely standardized values for coefficients and covariances. 
suggested in the factor-item structure by the modification indices. In addition, and in accordance with the theoretical framework, these three categories (factors) proved to be closely related with each other. Overall, this model presented a very good fit to the data, ${ }^{21-23}$ suggesting that it can be used to represent (and assess) MC.

In the second phase, the replication of the initially found model structure resulted also in a very good fit to the other half sample data (calibration sample), indicating a good reliability of the model to reproduce MC data. In the last step we formally tested for measure and structural invariance between the two half-split samples, in order to cross-validate for measurement and structural invariance. In both cases, the tested model showed a very adequate fit, concluding for its overall validity for interpreting MC in children and adolescents.

Therefore, our results postulate that MC can be advantageously represented by locomotor (SHR and SLJ), stability (moving platform and jumping laterally), and manipulative (kick and throw velocity) categories of movement skills, and that the latent essence of each of these categories can be objectively measured by two quantitative motor tasks. This model presents several advantages for research, education, and clinical settings.

The first advantage is the use of a set of motor tasks widely used in past research settings as representative of MC categories. ${ }^{11,16,20}$ The second advantage is the parsimony of the model. Unlike other models' protocols that use several motor tasks, such as the TGMD ${ }^{24}$ or $\mathrm{M}-\mathrm{ABC}^{25}$ or even some non-standardized protocols, ${ }^{13}$ our final model is only comprised by six feasible tests. The third advantage is that this model uses objective (quantitative) measures. Qualitative methods are focused on the process, providing insight into the form or characteristics of the movement; therefore, requiring a greater knowledge of the movement components and usually require a lot of time to analyze the data. Quantitative approaches are focused only on the final product and enable a faster assessment of the performance outcome with a high level of reliability over time. ${ }^{26}$ These methods are sensitive discriminators among competence levels across childhood and early adolescence, ${ }^{20}$ and are correlated with qualitative process-oriented assessments of the skills. ${ }^{27-29}$ Moreover, quantitative methods also do not require a high level of expertise and training of the evaluators, as usually recommended in qualitative methods, ${ }^{13}$ since the lack of subjectivity inherent to the quantitative approaches permits that even less experienced observers can apply it. The entire protocol takes about 10 min per participant; however children can be grouped in small groups, reducing the average time needed for assessment. Furthermore, the results information can be immediately used, making it a huge advantage for the use in PE classes, and sports' environments. The fourth advantage is that the motor tasks used do not have a ceiling effect over developmental years, and so the same model and protocol can be used from childhood to adult years. The fifth advantage is that the model, giving the magnitude of the correlations between factors, suggests the possibility to obtain a global composite score of MC, in addition to the categories' scores.

This model is representative of MC and can be used by researchers, PE teachers, and health and sports training professionals, in order to objectively monitor motor development. This MC model seems promising, but further research is warranted to replicate the current results.

This study has some limitations. The results confirmed the agreement of six of them with the tested model, nevertheless, and in order to achieve a more accurate representation of MC, a broader range of motor tests could be warranted in next studies. In addition, in order to be consistent with the number of trials for each skill, the use of three trials could be more appropriate to select the best score.
It is also important to note that our sample had children from 6 to 14 years old and the results might even have a better adjustment for separate groups of age and gender. Future investigations should take into consideration age and gender.

\section{Conclusion}

Our results support the idea that MC can be evaluated through a protocol with six motor tasks that represent the three major latent variables of MC (i.e., stability, locomotor and manipulative). We suggest that the use of a quantitative approach with few motor tasks without a celling effect, which are representative of the major MC components, is a good alternative to the existing testing protocols. Because the tested motor tasks are easy to assess, PE teachers or even trained classroom teachers can use this model regularly in their practices and evaluations.

\section{Practical implications}

- Brief and easy to administer evaluation model representative of MC, which can be used by several professionals to objectively monitor MC in several contexts.

- The teaching of motor skills should be integrated into the PE curriculum activities, and teachers could use this model protocol to assess children's MC.

- This regular assessment can help teachers to develop the best approach and exercises to improve their student's MC.

\section{Acknowledgments}

We would like to thank the schools, children and parents for their participation in this study. We also thank everyone who helped with the data collection.

\section{References}

1. Stodden David F, Goodway Jacqueline D, Langendorfer Stephen J et al. A developmental perspective on the role of motor skill competence in physical activity: an emergent relationship. Quest 2008; 60(2):290-306. http://dx.doi.org/10.1080/00336297.2008.10483582.

2. Fransen Job, D'Hondt Eva, Bourgois Jan et al. Motor competence assessment in children: convergent and discriminant validity between the BOT-2 Short Form and KTK testing batteries. Res Dev Disabil 2014; 35(6):1375-1383. http://dx.doi.org/10.1016/j.ridd.2014.03.011.

3. Lopes VP, Rodrigues LP, Maia JAR et al. Motor coordination as predictor of physical activity in childhood. Scand J Med Sci Sports 2011; 21(5):663-669. http://dx.doi.org/10.1111/j.1600-0838.2009.01027.x.

4. Holfelder Benjamin, Schott Nadja. Relationship of fundamental movement skills and physical activity in children and adolescents: a systematic review. Psychol Sport Exerc 2014; 15:382-391. http://dx.doi.org/10.1016/j.psychsport.2014.03.005.

5. Haga Monika. Physical fitness in children with high motor competence is different from that in children with low motor competence. Phys Ther 2009; 89(10):1089-1097. http://dx.doi.org/10.2522/ptj.20090052.

6. Vandendriessche Joric B, Vandorpe Barbara, Coelho-e-Silva Manuel J et al. Multivariate association among morphology, fitness, and motor coordination characteristics in boys age 7 to 11. Pediatr Exerc Sci 2011; 23(4):504-520.

7. Hands B, Larkin D, Parker $\mathrm{H}$ et al. The relationship among physical activity, motor competence and health-related fitness in 14-yearold adolescents. Scand J Med Sci Sports 2009; 19(5):655-663. http://dx.doi.org/10.1111/j.1600-0838.2008.00847.x.

8. Barnett Lisa M, Morgan Philip J, Beurden Eric et al. Perceived sports competence mediates the relationship between childhood motor skill proficiency and adolescent physical activity and fitness: a longitudinal assessment. Int J Behav Nutr Phys Act 2008; 5:40. http://dx.doi.org/10.1186/1479-5868-5-40.

9. Barnett Lisa M, Morgan Philip J, Van Beurden Eric et al. A reverse pathway? Actual and perceived skill proficiency and physical activity. Med Sci Sports Exerc 2011; 43:898-904. http://dx.doi.org/10.1249/MSS.0b013e3181fdfadd.

10. Lopes Vítor P, Stodden David F, Bianchi Mafalda M et al. Correlation between BMI and motor coordination in children. J Sci Med Sport 2012; 15(1):38-43. http://dx.doi.org/10.1016/j.jsams.2011.07.005. 
11. Gallahue David, Ozmun John, Goodway Jackie. Understanding motor development: infants, children, adolescents, adults, 7th ed New York, NY, McGraw-Hill, 2012.

12. Hume Clare, Okely Anthony, Bagley Sarah et al. Does weight status influence associations between children's fundamental movement skills and physical activity? Res Q Exerc Sport 2008; 79(2):158-165. http://dx.doi.org/10.1080/02701367.2008.10599479.

13. Okely AD, Booth ML. Mastery of fundamental movement skills among children in New South Wales: prevalence and sociodemographic distribution. J Sci Med Sport 2004:358-372

14. Schulz Joerg, Henderson Sheila E, Sugden David A et al. Structural validity of the Movement $\mathrm{ABC}-2$ test: factor structure comparisons across three age groups. Res Dev Disabil 2011; 32(4):1361-1369. http://dx.doi.org/10.1016/j.ridd.2011.01.032.

15. Kim Seonjin, Kim Min Joo, Valentini Nadia $C$ et al. Validity and reliability of the TGMD-2 for South Korean children. J Mot Behav 2014; 46(5):351-356. http://dx.doi.org/10.1080/00222895.2014.914886.

16. Ernst Kiphard, Schilling F. Körperkoordinationstest für Kinder: KTK Manual, Göttingen, Beltz Test, 2007.

17. Vicente-Rodríguez Germán, Rey-López Juan $\mathrm{P}$, Ruíz Jonathan $\mathrm{R}$ et al. Interrater reliability and time measurement validity of speed-agility field tests in adolescents. J Strength Cond Res 2011; 25(7):2059-2063. http://dx.doi.org/10.1519/JSC.0b013e3181e742fe.

18. Ara Ignacio, Moreno Luis, Leiva Maria T et al. Adiposity, physical activity, and physical fitness among children from Aragón, Spain. Obesity (Silver Spring) 2007; 15(8):1918-1924. http://dx.doi.org/10.1038/oby.2007.228.

19. Beashel Paul. AQA Sport Examined, Cheltenham, Nelson Thornes, 2004
20. Stodden David F, Gao Z, Langendorfer SJ et al. Dynamic relationships between motor skill competence and health-related fitness in youth. Pediatr Exerc Sc 2014; 26(3):231-241. http://dx.doi.org/10.1123/pes.2013-0027.

21. Byrne Barbara. Structural Equation Modeling with EQS: Basic Concepts, Applications, and Programming, Mahwah, NJ, Lawrence Erlbaum Associates, 2006.

22. Bentler PM. EQS 6 Structural Equations Program Manual, Encino, CA, Multivariate Software, Inc, 2006

23. Raykov Tenko, Marcoulides George A. A First Course in Structural Equation Modeling, Mahwah, NJ, L. Erlbaum Associates, 2006.

24. Ulrich DA. Test of Gross Motor Development, Austin, TX, Pro-ED. Inc, 1985.

25. Henderson SE, Sugden DA. Movement Assessment Battery for Children Manual, London, The Psychological Corporation Ltd, 1992.

26. Spray Judith A. Recent developments in measurement and possible applications to the measurement of psychomotor behavior. Res Q Exerc Sport 1987; 58(3):203-209. http://dx.doi.org/10.1080/02701367.1987.10605449.

27. Mally Kristi $K$, Battista Rebecca A, Roberton Mary Ann. Distance as a control parameter for place kicking. J Hum Sport Exerc 2011; 6(1):122-134. http://dx.doi.org/10.4100/jhse.2011.61.14.

28. Stodden David F, Langendorfer Stephen J, Fleisig Glenn S et al. Kinematic constraints associated with the acquisition of overarm throwing part II: Upper extremity actions. Res Q Exerc Sport 2006; 77(4):428-436. http://dx.doi.org/10.5641/027013606X13080770015166.

29. Stodden David F, Langendorfer Stephen J, Fleisig GS et al. Kinematic constraints associated with the acquisition of overarm throwing, part I: Upper extremity actions. Res Q Exerc Sport 2006; 77(4):417-427. http://dx.doi.org/10.1080/02701367.2006.10599377. 